\title{
A nonlocal reaction-diffusion model for a single species with stage structure and distributed maturation delay
}

\author{
J. F. M. AL-OMARI ${ }^{1}$ and S. A. GOURLEY ${ }^{2}$ \\ ${ }^{1}$ Faculty of Engineering Technology, PO Box 15008, Al-Balqa Applied University, Amman 11134, Jordan \\ ${ }^{2}$ Department of Mathematics and Statistics, University of Surrey, Guildford, Surrey GU2 $7 X H, U K$ \\ e-mail: s.gourley@surrey.ac.uk
}

(Received 14 January 2004; revised 26 August 2004)

\begin{abstract}
We propose a delay differential equation model for a single species with stage-structure in which the maturation delay is modelled as a distribution, to allow for the possibility that individuals may take different amounts of time to mature. General birth and death rate functions are used. We find that the dynamics of the model depends largely on the qualitative form of the birth function, which depends on the total number of adults. If it is monotonic increasing and a non-zero equilibrium exists, then the equilibrium is globally stable for all maturation delay distributions with compact support. For the case of a finite spatial domain with impermeable boundaries, a reaction-diffusion extension of the model is rigorously derived using an approach based on the von Foerster diffusion equation. The resulting reaction-diffusion system is nonlocal. The dynamics of the reaction-diffusion system again depends largely on the qualitative form of the birth function. If the latter is nonmonotone with a single hump, then the dynamics depends largely on whether the equilibrium is to the left or right of the hump, with oscillatory dynamics a possibility if it is sufficiently far to the right.
\end{abstract}

\section{Introduction}

The following system for a single species population was proposed by Aiello \& Freedman [1]:

$$
\begin{aligned}
& u_{i}^{\prime}(t)=\alpha u_{m}(t)-\gamma u_{i}(t)-\alpha e^{-\gamma \tau} u_{m}(t-\tau), \\
& u_{m}^{\prime}(t)=\alpha e^{-\gamma \tau} u_{m}(t-\tau)-\beta u_{m}^{2}(t),
\end{aligned}
$$

where $\alpha, \beta, \gamma$ and the delay $\tau$ are positive constants. In this system, $u_{i}$ and $u_{m}$ denote the numbers of immature (juvenile) and mature (adult) members of the population respectively, and the delay $\tau$ is the time taken from birth to maturity. System (1.1) provides an alternative, and arguably more realistic, model for a single population than the well known logistic equation $u^{\prime}=u(1-u)$ by recognising that individuals cannot reproduce right from birth but only after they have become mature, which takes time $\tau$. It is assumed that the birth rate at any time is proportional to the number of adults at that time, so that the birth rate at time $t$ is $\alpha u_{m}(t)$. The first term in the right hand side of the second equation of (1.1) represents the rate of recruitment into the adult population. 
This rate is essentially the birth rate $\tau$ time units ago, discounted by the factor $e^{-\gamma \tau}$ which accounts for mortality during the juvenile phase. The parameter $\gamma$ measures the juvenile death rate while $\beta$ measures the adult death rate. As shown in Aiello \& Freedman [1], all solutions of (1.1), other than the trivial solution, converge to the equilibrium solution

$$
\left(u_{i}, u_{m}\right) \equiv\left(\frac{\alpha^{2}}{\beta \gamma} e^{-\gamma \tau}\left(1-e^{-\gamma \tau}\right), \frac{\alpha}{\beta} e^{-\gamma \tau}\right) .
$$

Aiello and Freedman assume that the maturation delay $\tau$ is known exactly and that all individuals take this amount of time to mature. The aims of the present paper are threefold. First, we aim to modify (1.1) to allow for the fact that the time from birth to maturity may be rather imperfectly known, or it might vary from individual to individual. Secondly, we shall allow a more general birthrate function and a more general deathrate for the matures. Thirdly, we shall consider how to incorporate spatial effects into the resulting system of equations, and we shall show that these result in a nonlocal reaction-diffusion system which we shall analyse using comparison principles.

We will still assume that the birth rate at any time is related to the number of adults at that time, but the birth rate in this paper will be $b\left(u_{m}(t)\right)$ rather than $\alpha u_{m}(t)$, where $b(\cdot)$ is called the births function and satisfies certain assumptions. Two cases of particular interest are the case when $b\left(u_{m}\right)$ is increasing for all $u_{m}>0$, and the case when $b\left(u_{m}\right)$ qualitatively resembles $P u_{m} e^{-A u_{m}}$ which is particularly relevant in modelling populations which cannot reproduce if two many individuals are present. We shall leave the death rate for the immatures as $\gamma u_{i}(t)$, but for the matures we shall replace $\beta u_{m}^{2}(t)$ by a more general death rate function $d\left(u_{m}(t)\right)$ satisfying certain assumptions.

Replacing $\alpha u_{m}(t)$ by $b\left(u_{m}(t)\right)$ has the consequence that the adult recruitment term in $(1.1)$ becomes $e^{-\gamma \tau} b\left(u_{m}(t-\tau)\right)$, but we now want to address the point about uncertainty in the maturation delay. We propose to replace $e^{-\gamma \tau} b\left(u_{m}(t-\tau)\right)$ by a distributed delay term allowing for a distribution of maturation times, weighted by a probability density function (the function $f$ appearing below). We shall assume that at time $t$ the number that become mature, per unit time, is

$$
\int_{0}^{\infty} b\left(u_{m}(t-s)\right) f(s) e^{-\gamma s} d s
$$

The term $b\left(u_{m}(t-s)\right)$ is the birth rate at time $t-s$, assuming that the birth rate at a given time is a function of the number of mature adults around at that time. The quantity $f(s) d s$, with $d s$ infinitesimal, is the probability that an individual member takes an amount of time between $s$ and $s+d s$ to mature, and $e^{-\gamma s}$ is the probability of an individual born at time $t-s$ still being alive at time $t$. Individuals becoming mature at time $t$ could have been born at any time prior to this, and the integral totals up the contributions from all previous times. Therefore, in the absence of any spatial effects the model (1.1) is generalised to

$$
\begin{aligned}
& u_{i}^{\prime}(t)=b\left(u_{m}(t)\right)-\gamma u_{i}(t)-\int_{0}^{\infty} b\left(u_{m}(t-s)\right) f(s) e^{-\gamma s} d s, \\
& u_{m}^{\prime}(t)=\int_{0}^{\infty} b\left(u_{m}(t-s)\right) f(s) e^{-\gamma s} d s-d\left(u_{m}(t)\right),
\end{aligned}
$$

where $\int_{0}^{\infty} f(s) d s=1$ and $f \geqslant 0$, because $f$ is a probability density function. System (1.1) is a particular case of system (1.2). It arises when we take $f(s)=\delta(s-\tau)$, where $\delta$ is the Dirac delta function, $b\left(u_{m}\right)=\alpha u_{m}$ and $d\left(u_{m}\right)=\beta u_{m}^{2}$. 


\section{Positivity}

We will prove the following theorem.

Theorem 1 In system (1.2), assume that $b(0)=0$ and $b\left(u_{m}\right)>0$ when $u_{m}>0$. Assume also that $d(0)=0$. Let $\phi$ denote the initial data for $u_{m}$, so that $u_{m}(t)=\phi(t)$ for all $t \in(-\infty, 0]$. Let $\phi$ be continuous and satisfy $\phi(t) \geqslant 0$ for all $t \in(-\infty, 0)$, and $\phi(0)>0$. Then $u_{m}(t), u_{i}(t)>0$ for all $t>0$.

Proof We first show that $u_{m}(t)>0$ for all $t \in(0, \infty)$. Assume, for contradiction, that there exists a first time $t_{0}>0$ such that $u_{m}\left(t_{0}\right)=0$. Then $u_{m}(t)>0$ for $t \in\left[0, t_{0}\right)$ and

$$
\begin{aligned}
u_{m}^{\prime}\left(t_{0}\right) & =\int_{0}^{\infty} b\left(u_{m}\left(t_{0}-s\right)\right) f(s) e^{-s \gamma} d s \\
& =\int_{0}^{t_{0}} b\left(u_{m}\left(t_{0}-s\right)\right) f(s) e^{-s \gamma} d s+\int_{t_{0}}^{\infty} b\left(u_{m}\left(t_{0}-s\right)\right) f(s) e^{-s \gamma} d s \\
& =\int_{0}^{t_{0}} \underbrace{b\left(u_{m}\left(t_{0}-s\right)\right)}_{>0} f(s) e^{-s \gamma} d s+\int_{t_{0}}^{\infty} \underbrace{b\left(\phi\left(t_{0}-s\right)\right)}_{\geqslant 0} f(s) e^{-s \gamma} d s \\
& >0 .
\end{aligned}
$$

Thus, $u_{m}^{\prime}\left(t_{0}\right)>0$. This is a contradiction since it implies $u_{m}(t)$ must be negative for $t$ just before $t_{0}$, which contradicts the choice of $t_{0}$.

Proving that $u_{i}(t)>0$ for $t>0$ is a little more difficult. We shall first calculate the total number of individuals that are immature at time $t=0$ (i.e. the initial condition for $u_{i}$ ). This will be composed of all individuals born at times $s<0$ who are still alive at $t=0$ and have not yet matured by this time. For $s \in(-\infty, 0]$ the birth rate of immatures $u_{i}(s)$ is $b(\phi(s))$. So, for a typical $s<0$, the number of births between times $s$ and $s+d s$ is $b(\phi(s)) d s$, for small $d s$. The number of these individuals still alive at time $t=0$ will be $b(\phi(s)) e^{-\gamma(0-s)} d s$ or $b(\phi(s)) e^{\gamma s} d s$. However, some of them may have already matured and we need to remove these from the count. Now, since $f(\xi) d \xi$ is the probability of becoming mature between age $\xi$ and $\xi+d \xi$ (for $d \xi$ small), the probability of an individual born at a particular time $s<0$ having become mature before time $t=0$ will be $\int_{0}^{-s} f(\xi) d \xi$ (recall $s<0$ ). Therefore, the fraction of the surviving individuals born at time $s<0$ that are still immature at time $t=0$ is $1-\int_{0}^{-s} f(\xi) d \xi$, or $\int_{-s}^{\infty} f(\xi) d \xi$. So, $\left(\int_{-s}^{\infty} f(\xi) d \xi\right) b(\phi(s)) e^{\gamma s} d s$ is the number of those born at time $s<0$ that are still alive and still immature at time $t=0$. To total up the births from all negative times we integrate over $s$, obtaining

$$
u_{i}(0)=\int_{-\infty}^{0}\left(\int_{-s}^{\infty} f(\xi) d \xi\right) b(\phi(s)) e^{\gamma s} d s
$$

In system (1.2) the initial conditions for $u_{i}$ and $u_{m}$ are not independent of each other. The initial value for $u_{i}$ is related to the initial data for $u_{m}$ through expression (2.1). We will now prove that the solution $u_{i}(t)$ of the first equation of system (1.2), subject to the initial condition (2.1), is strictly positive for all $t>0$. In fact, we shall do this by showing that 
the solution is given explicitly by

$$
u_{i}(t)=\int_{-\infty}^{t}\left(\int_{t-s}^{\infty} f(\xi) d \xi\right) b\left(u_{m}(s)\right) e^{-\gamma(t-s)} d s,
$$

which is strictly positive, since we have already shown $u_{m}(t)$ is positive.

Obviously $u_{i}(t)$ given by (2.2) satisfies the initial condition (2.1). We need to show that (2.2) satisfies the differential equation for $u_{i}$, the first equation of system (1.2). But

$$
\begin{aligned}
\frac{d u_{i}}{d t}= & \int_{-\infty}^{t}\left\{\left(\int_{t-s}^{\infty} f(\xi) d \xi\right)\left(-\gamma b\left(u_{m}(s)\right) e^{-\gamma(t-s)}\right)-f(t-s) b\left(u_{m}(s)\right) e^{-\gamma(t-s)}\right\} d s \\
& +\underbrace{\left(\int_{0}^{\infty} f(\xi) d \xi\right)}_{=1} b\left(u_{m}(t)\right) \\
= & -\gamma u_{i}(t)+b\left(u_{m}(t)\right)-\int_{-\infty}^{t} f(t-s) b\left(u_{m}(s)\right) e^{-\gamma(t-s)} d s \\
= & -\gamma u_{i}(t)+b\left(u_{m}(t)\right)-\int_{0}^{\infty} f(s) b\left(u_{m}(t-s)\right) e^{-\gamma s} d s,
\end{aligned}
$$

so that the differential equation is satisfied.

\section{Monotone birth functions}

This section considers the case when the birth function $b\left(u_{m}\right)$ in system (1.2) is increasing for all $u_{m}>0$. We will assume that the kernel $f(s)$ has compact support, that is, $f(s)=0$ for $s \geqslant \tau$ for some $\tau>0$. This implies that no individual ever takes longer than $\tau$ units of time to become mature. We still assume the kernel is normalised (see (A1) below).

Where we are interested in monotone birth functions, our assumptions may be summed up as:

The kernel $f$ satisfies $f(s)=0$ for all $s \geqslant \tau$, for some $\tau>0$, and $\int_{0}^{\tau} f(s) d s=1$. Also, $b(0)=0$ and $b\left(u_{m}\right)$ is strictly increasing for

(A1) : $\quad$ all $u_{m}>0$, and $d(0)=0$. Moreover, (3.1) has a root $\hat{u}_{m}>0$ with $b\left(u_{m}\right) \int_{0}^{\tau} f(s) e^{-\gamma s} d s>d\left(u_{m}\right)$ when $0<u_{m}<\hat{u}_{m}$ and $b\left(u_{m}\right) \int_{0}^{\tau} f(s) e^{-\gamma s}$ $d s<d\left(u_{m}\right)$ when $u_{m}>\hat{u}_{m}$.

Since $b(0)=d(0)=0,\left(u_{i}, u_{m}\right)=(0,0)$ is an equilibrium of (1.2). The $u_{m}$ component $\hat{u}_{m}$ of any other equilibrium has to satisfy

$$
b\left(\hat{u}_{m}\right) \int_{0}^{\tau} f(s) e^{-\gamma s} d s=d\left(\hat{u}_{m}\right) .
$$

Existence of a root $\hat{u}_{m}>0$ of (3.1) holds under (A1). In this case the $u_{i}$ component $\hat{u}_{i}$ of the equilibrium is given by

$$
\hat{u}_{i}=\frac{b\left(\hat{u}_{m}\right)}{\gamma}\left(1-\int_{0}^{\tau} f(s) e^{-\gamma s} d s\right)>0 .
$$

We will prove the following theorem. 
Theorem 2 Let (A1) hold, and assume that $u_{m}(t) \geqslant 0$ for $-\tau \leqslant t<0$ and $u_{m}(0)>0$. Then $u_{m}(t) \rightarrow \hat{u}_{m}$ and $u_{i}(t) \rightarrow \hat{u}_{i}$ as $t \rightarrow \infty$, where $\hat{u}_{m}$ and $\hat{u}_{i}$ are given by (3.1) and (3.2), respectively.

Remark For ecological realism $u_{i}(0)$ should be given by (2.1). However Theorem 2 can be proved without any restriction on $u_{i}(0)$.

Proof The proof is in several stages. We first prove that the solution $u_{m}(t)$ of

$$
u_{m}^{\prime}(t)=\int_{0}^{\tau} b\left(u_{m}(t-s)\right) f(s) e^{-\gamma s} d s-d\left(u_{m}(t)\right)
$$

satisfies $u_{m}(t) \rightarrow \hat{u}_{m}$ and will first consider the case in which $u_{m}(t)$ is eventually monotone (i.e. monotone for all $t$ sufficiently large). It is easy to see that $u_{m}(t)$ cannot be eventually monotonic increasing and go to $+\infty$ since otherwise (3.3) yields, for all $t$ sufficiently large,

$$
u_{m}^{\prime}(t)=\int_{0}^{\tau} b\left(u_{m}(t-s)\right) f(s) e^{-\gamma s} d s-d\left(u_{m}(t)\right) \leqslant b\left(u_{m}(t)\right) \int_{0}^{\tau} f(s) e^{-\gamma s} d s-d\left(u_{m}(t)\right)<0 .
$$

Thus if $u_{m}(t)$ is eventually monotonic increasing then it must tend to a limit. If $u_{m}(t)$ is eventually monotonic decreasing then, by positivity of solutions, $u_{m}(t)$ must approach a limit but in principle the limit could be zero and this is what we must rule out next. Suppose, for contradiction, that $u_{m}(t) \rightarrow 0$ and let $t_{0}$ be sufficiently large that, for all $t \geqslant t_{0}$, $u_{m}(t)<\hat{u}_{m}$. Let

$$
\mu=\min _{t \in\left[t_{0}, t_{0}+\tau\right]} u_{m}(t)
$$

Then $\mu<\hat{u}_{m}$ and, by Theorem $1, \mu>0$. To obtain a contradiction it suffices to show that $u_{m}(t)>\mu$ for all $t \geqslant t_{0}+\tau$. If the latter were false then there would exist a $t^{*} \geqslant t_{0}+\tau$ such that $u_{m}\left(t^{*}\right)=\mu, u_{m}(t) \geqslant \mu$ for all $t \in\left[t^{*}-\tau, t^{*}\right]$ and $u_{m}^{\prime}\left(t^{*}\right) \leqslant 0$. But then (3.3) gives

$$
u_{m}^{\prime}\left(t^{*}\right)=\int_{0}^{\tau} b\left(u_{m}\left(t^{*}-s\right)\right) f(s) e^{-\gamma s} d s-d(\mu) \geqslant b(\mu) \int_{0}^{\tau} f(s) e^{-\gamma s} d s-d(\mu)>0
$$

since $\mu<\hat{u}_{m}$. Thus $u_{m}(t)$ cannot approach zero. As a result of what has been ruled out above, we must now conclude that any eventually monotone solution approaches a nonzero limit, say $\bar{u}_{m}$. This limit must be an equilibrium of (3.3) and so, since $\bar{u}_{m}>0$, we must have $\bar{u}_{m}=\hat{u}_{m}$.

We now consider the case when $u_{m}(t)$ is not eventually monotonic. Of the various possibilities which then arise, it will be sufficient for us to consider the case when the graph of $u_{m}(t)$ has an infinite sequence of maxima greater than $\hat{u}_{m}$ (other cases can be dealt with similarly). So, there exists a sequence of times $\left\{t_{j}\right\}, j=1,2, \ldots$ such that $t_{j} \rightarrow \infty$ as $j \rightarrow \infty, t_{1} \geqslant 2 \tau, u_{m}\left(t_{j}\right)>\hat{u}_{m}, u_{m}^{\prime}\left(t_{j}\right)=0$ and $u_{m}^{\prime \prime}\left(t_{j}\right)<0$.

We claim that $\sup _{t \geqslant t_{1}} u_{m}(t)=u_{m}\left(t_{k}\right)$, for some integer $k$. For if not, then there exists a subsequence, still denoted $\left\{t_{j}\right\}$, such that $u_{m}\left(t_{j}\right)>u_{m}(t)$ for $2 \tau<t<t_{j}, t_{j} \rightarrow \infty$ as $j \rightarrow \infty$. This is because, if our claim is not true, then beyond every local maximum of $u_{m}(t)$ there must be another that is even higher. The subsequence is generated by including each 
maximum that is higher than every maximum before it. Then, from (3.3),

$$
u_{m}^{\prime}\left(t_{j}\right)<b\left(u_{m}\left(t_{j}\right)\right) \int_{0}^{\tau} f(s) e^{-\gamma s} d s-d\left(u_{m}\left(t_{j}\right)\right)<0,
$$

since $u_{m}\left(t_{j}\right)>\hat{u}_{m}$. This is a contradiction since $u_{m}^{\prime}\left(t_{j}\right)=0$.

Now let $s_{1}=t_{k}$. By the same arguments applied to the interval $t \geqslant t_{k+1}$ we can find a $t_{l}(l>k)$ such that $\sup _{t \geqslant t_{k+1}} u_{m}(t)=u_{m}\left(t_{l}\right)$. Let $s_{2}=t_{l}$. We can continue this process to find $t_{n}(n>l)$ such that $\sup _{t \geqslant t_{l+1}} u_{m}(t)=u_{m}\left(t_{n}\right)$. Similarly, we define $s_{3}, s_{4}, \ldots$, the sequence $\left\{s_{j}\right\}$ having the property that $s_{j}<s_{j+1}, s_{j} \rightarrow \infty$ as $j \rightarrow \infty$ and $u_{m}\left(s_{j}\right)>u_{m}(t)$ for all $t>s_{j}$ (in particular $u_{m}\left(s_{j}\right)>u_{m}\left(s_{j+1}\right)$ ). Also, $u_{m}^{\prime}\left(s_{j}\right)=0$ and each $u_{m}\left(s_{j}\right)>\hat{u}_{m}$. These observations imply that $\alpha_{j}:=u_{m}\left(s_{j}\right)$ approaches a limit $\alpha$ as $j \rightarrow \infty$, with $\alpha \geqslant \hat{u}_{m}$, and we have to show that $\alpha=\hat{u}_{m}$.

From the sequence $\left\{s_{j}\right\}$ choose a subsequence, again denoted $\left\{s_{j}\right\}$, such that $s_{j}-\tau \geqslant s_{j-1}$. From (3.3) evaluated at $t=s_{j}$, and the fact that $u_{m}(t) \leqslant u_{m}\left(s_{j-1}\right)$ for $t \geqslant s_{j-1}$, we have

$$
\begin{aligned}
d\left(\alpha_{j}\right) & =\int_{0}^{\tau} b\left(u_{m}\left(s_{j}-s\right)\right) f(s) e^{-\gamma s} d s \\
& \leqslant b\left(\alpha_{j-1}\right) \int_{0}^{\tau} f(s) e^{-\gamma s} d s .
\end{aligned}
$$

Letting $j \rightarrow \infty$ gives $d(\alpha) \leqslant b(\alpha) \int_{0}^{\tau} f(s) e^{-\gamma s} d s$ which, from the hypotheses of the theorem, implies $\alpha \leqslant \hat{u}_{m}$. Together with $\alpha \geqslant \hat{u}_{m}$, this implies $\alpha=\hat{u}_{m}$. We have shown that $\lim _{t \rightarrow \infty} u_{m}(t)=\hat{u}_{m}$.

To complete the proof we need to show that $\lim _{t \rightarrow \infty} u_{i}(t)=\hat{u}_{i}$. This is straightforward. In view of the assumption of $f(s)$ having compact support, the first equation of (1.2), as $t \rightarrow \infty$, becomes

$$
u_{i}^{\prime}(t)=-\gamma u_{i}(t)+b\left(\hat{u}_{m}\right)\left(1-\int_{0}^{\tau} f(s) e^{-\gamma s} d s\right)
$$

and therefore $u_{i}(t) \rightarrow \hat{u}_{i}$, given by (3.2).

This argument can be made rigorous by using the theory of asymptotically autonomous differential equations (see, for example, Mischaikow et al. [4]).

\section{The effect of diffusion}

In this section we study the effect of adding diffusion terms to system (1.2) to model the situation when individuals can drift around. We shall take the diffusion to be of the simplest reasonable kind for models in continuous space which is Fickian diffusion. In this representation of diffusion the individuals are performing a random walk and, for a model without delay, the diffusion would be modelled by adding Laplacian terms to the right hand sides of the equations to obtain a reaction-diffusion system. When delays are present the situation is a little more complicated because the individuals may have moved during the period of the time delay (the maturation delay, in our case). In consequence, the time delay terms in the resulting system assume a somewhat more complicated appearance. In a finite $N$ dimensional spatial domain $\Omega$ with impermeable boundaries, we shall argue that if $D_{i}$ and $D_{m}$ denote the diffusivities of the immature and mature members respectively, 
then an appropriate reaction diffusion extension of system (1.2) is

$$
\begin{aligned}
& \frac{\partial u_{i}(x, t)}{\partial t}=D_{i} \Delta u_{i}(x, t)+b\left(u_{m}(x, t)\right)-\gamma u_{i}(x, t)-\int_{0}^{\tau} \int_{\Omega} G(x, y, s) f(s) e^{-\gamma s} b\left(u_{m}(y, t-s)\right) d y d s, \\
& \frac{\partial u_{m}(x, t)}{\partial t}=D_{m} \Delta u_{m}(x, t)+\int_{0}^{\tau} \int_{\Omega} G(x, y, s) f(s) e^{-\gamma s} b\left(u_{m}(y, t-s)\right) d y d s-d\left(u_{m}(x, t)\right),
\end{aligned}
$$

for $x \in \Omega \subset \mathbf{R}^{N}, t>0$, subject to the homogeneous Neumann boundary conditions

$$
\mathbf{n} \cdot \nabla u_{i}=\mathbf{n} \cdot \nabla u_{m}=0 \quad \text { on } \partial \Omega
$$

where $\mathbf{n}$ is an outward pointing normal to $\partial \Omega$. The initial conditions for (4.1) are

$$
u_{i}(x, 0)=u_{i}^{0}(x), \quad u_{m}(x, s)=u_{m}^{0}(x, s), \quad(x, s) \in \bar{\Omega} \times[-\tau, 0]
$$

where $u_{i}^{0}$ and $u_{m}^{0}$ are prescribed. In system $(4.1), G(x, y, t)$ is the solution, subject to homogeneous Neumann boundary conditions, of

$$
\frac{\partial G}{\partial t}=D_{i} \Delta_{x} G, \quad G(x, y, 0)=\delta(x-y)
$$

where $\delta$ is the Dirac delta function, and where $\Delta_{x}$ means the Laplacian computed with respect to the first argument of $G(x, y, t)$ (recall that $x$ is a vector in $\mathbf{R}^{N}$ here), and $y \in \mathbf{R}^{N}$ is for these purposes a parameter. It is easy to see that

$$
\int_{\Omega} G(x, y, t) d x=\int_{\Omega} G(x, y, t) d y=1
$$

for all $t \geqslant 0$. Note also that the second equation of (4.1) involves both of the two diffusivities $D_{i}$ and $D_{m}$ since the kernel $G$ involves $D_{i}$.

Let us now derive the term with the double integral (the adult recruitment term) in the right hand side of the second equation of (4.1), assuming that the birth rate at a particular location and time is some function of the number of adults at that point in space at that time. Let $u(x, t, a)$ be the density of individuals of age $a$ at location $x \in \Omega$ at time $t$. Fix $s>0$. At $(x, t)$ we want to calculate the rate of adult recruitment, i.e. the rate at which individuals are just reaching maturity. Of all the individuals just reaching maturity, some will have taken time $s$ to mature (more precisely, an amount of time between $s$ and $s+d s$ with $d s$ infinitesimal). Let us look at these individuals first. They will have been born at various locations in $\Omega$ and will have drifted around, being at point $x$ on becoming mature. During their juvenile phase let us assume they evolved according to the von Foerster type equation

$$
\frac{\partial u}{\partial t}+\frac{\partial u}{\partial a}=D_{i} \Delta u-\gamma u, \quad x \in \Omega, \quad 0<a<s
$$

with homogeneous Neumann boundary conditions, and subject to

$$
u(x, t, 0)=b\left(u_{m}(x, t)\right)
$$

where $b$ is the birth function. Our aim at this stage is to find an expression for $u(x, t, s)$ in terms of $u_{m}$. 
Let $v(x, r, a)=u(x, a+r, a)$. Then

$$
\begin{aligned}
\frac{\partial v}{\partial a} & =\left[\frac{\partial u}{\partial t}+\frac{\partial u}{\partial a}\right]_{t=a+r}=\left[D_{i} \Delta u-\gamma u\right]_{t=a+r} \\
& =D_{i} \Delta u(x, a+r, a)-\gamma u(x, a+r, a)
\end{aligned}
$$

so that

with

$$
\frac{\partial v}{\partial a}=D_{i} \Delta v-\gamma v, \quad x \in \Omega, \quad a>0
$$

$$
v(x, r, 0)=b\left(u_{m}(x, r)\right) .
$$

The solution of (4.3) subject to (4.4) is

$$
v(x, r, a)=e^{-\gamma a} \int_{\Omega} G(x, y, a) b\left(u_{m}(y, r)\right) d y
$$

where the kernel $G$ is the function described above. Hence

$$
u(x, t, s)=e^{-\gamma s} \int_{\Omega} G(x, y, s) b\left(u_{m}(y, t-s)\right) d y .
$$

The above quantity gives us the rate, at $(x, t)$, at which individuals are becoming mature having taken time $s$ to mature. The total rate, at $(x, t)$, at which individuals become mature is

$$
\int_{0}^{\tau} u(x, t, s) f(s) d s
$$

or

$$
\int_{0}^{\tau} \int_{\Omega} G(x, y, s) f(s) e^{-\gamma s} b\left(u_{m}(y, t-s)\right) d y d s .
$$

Reaction-diffusion systems with delay have been of interest to investigators for around twenty years now. Systems similar to (4.1), but without the integral with respect to $y$, were studied by Yamada [6,7]. Reaction-diffusion systems which have both delay and nonlocal spatial effects are more recent. The results we shall prove in this paper utilise comparison principles due to Martin \& Smith [3] which have been developed in abstract settings that allow both time delay and spatial integration.

In the rest of this section, we prove theorems on the dynamics of system (4.1), considering separately the cases when the birth function is always increasing, and the case of a birth function $b\left(u_{m}\right)$ that qualitatively resembles $P u_{m} e^{-A u_{m}}$ with $A, P$ positive parameters. This particular case is of some importance in modelling certain insect populations [2]. We will restrict attention to kernels $f(s)$ with compact support.

\subsection{Monotone birth functions}

We will prove Theorem 3. Note that in the theorem there are no restrictions on the initial data for $u_{i}$. However, one can infer, using considerations similar to those that led to the expression for $u_{i}(0)$ in $\S 2$, that for biological realism the initial data for $u_{i}$ has to be related 
to that for $u_{m}$ by

$$
u_{i}^{0}(x)=\int_{-\tau}^{0} \int_{\Omega}\left\{\int_{-s}^{\tau} f(\xi) d \xi\right\} b\left(u_{m}^{0}(y, s)\right) e^{\gamma s} G(x, y,-s) d y d s
$$

although this is not necessary for Theorem 3 to hold.

Theorem 3 Let (A1) hold, and let the initial data for system (4.1) satisfy $u_{m}^{0}(x, s) \geqslant 0$ for all $(x, s) \in \bar{\Omega} \times[-\tau, 0]$ with $u_{m}^{0}(x, 0) \neq 0$. Then the solution of $(4.1),(4.2)$ satisfies

$$
\left(u_{i}(x, t), u_{m}(x, t)\right) \rightarrow\left(\hat{u}_{i}, \hat{u}_{m}\right)
$$

as $t \rightarrow \infty$, uniformly for $x \in \bar{\Omega}$, where $\hat{u}_{i}$ and $\hat{u}_{m}$ are given by (3.2) and (3.1).

Proof We shall first show that $u_{m}(x, t) \rightarrow \hat{u}_{m}$. The second equation of (4.1) involves $u_{m}$ only. Note also that the kernel $G$ in (4.1) is non-negative, as it is defined as the solution of the heat equation with non-negative initial data. In view of these facts, and the fact that $b\left(u_{m}\right)$ is increasing, a comparison principle holds for the second equation of (4.1). More precisely, if $u(x, t)$ and $w(x, t)$ are two functions satisfying the homogeneous Neumann boundary conditions, the inequality

$$
\begin{aligned}
& \frac{\partial u(x, t)}{\partial t}-D_{m} \Delta u(x, t)+d(u(x, t))-\int_{0}^{\tau} \int_{\Omega} G(x, y, s) f(s) e^{-\gamma s} b(u(y, t-s)) d y d s \\
& \leqslant \frac{\partial w(x, t)}{\partial t}-D_{m} \Delta w(x, t)+d(w(x, t))-\int_{0}^{\tau} \int_{\Omega} G(x, y, s) f(s) e^{-\gamma s} b(w(y, t-s)) d y d s
\end{aligned}
$$

for $t>0, x \in \Omega$, and the inequality $u(x, s) \leqslant w(x, s)$ for $(x, s) \in \bar{\Omega} \times[-\tau, 0]$, then

$$
u(x, t) \leqslant w(x, t) \quad \text { in } \bar{\Omega} \times(0, \infty) .
$$

This result follows from the work of Martin \& Smith [3]. The requirement that $u$ and $w$ should satisfy the homogeneous Neumann boundary requirements can in fact be relaxed but this fact is not helpful for our purposes here. By taking $u(x, t)$ to be $u_{m}(x, t)$, the solution of the second equation of (4.1) subject to (4.2), and $w(x, t)$ independent of $x$ and equal to $w(t)$ where

$$
\begin{aligned}
& w^{\prime}(t)=\int_{0}^{\tau} b(w(t-s)) f(s) e^{-s \gamma} d s-d(w(t)), \\
& w(s)=\max _{x \in \bar{\Omega}} u_{m}(x, s), \quad s \in[-\tau, 0],
\end{aligned}
$$

we have $u_{m}(x, t) \leqslant w(t)$ in $\bar{\Omega} \times(0, \infty)$. But the limiting behaviour of $w(t)$ is known from Theorem 2. Thus

$$
\limsup _{t \rightarrow \infty} \max _{x \in \bar{\Omega}} u_{m}(x, t) \leqslant \lim _{t \rightarrow \infty} w(t)=\hat{u}_{m} .
$$

If we can also show that

$$
\liminf _{t \rightarrow \infty} \min _{x \in \bar{\Omega}} u_{m}(x, t) \geqslant \hat{u}_{m},
$$


then we will have established that $u_{m}(x, t) \rightarrow \hat{u}_{m}$ uniformly in $x$. Proving (4.7) is similar to proving (4.6), but in the comparison principle we now take $w(x, t)$ as the solution $u_{m}(x, t)$ of the second equation of (4.1) subject to (4.2), and $u(x, t)$ independent of $x$ and equal to $u(t)$, the solution of

$$
\begin{aligned}
& u^{\prime}(t)=\int_{0}^{\tau} b(u(t-s)) f(s) e^{-s \gamma} d s-d(u(t)), \quad t>0, \\
& u(s)=\min _{x \in \bar{\Omega}} u_{m}(x, s), \quad s \in[-\tau, 0] .
\end{aligned}
$$

Again we invoke Theorem 2 to deduce the limiting behaviour of $u(t)$, and (4.7) follows immediately. The only possible problem here is that the minimum in (4.8) could be zero for all $s \in[-\tau, 0]$ without violating the assumptions on the initial data stated in the Theorem (if this were the case then $u(t)$ would be zero). However, our assumptions on $u_{m}^{0}$ and the strong maximum principle imply that $u_{m}(x, t)>0$ for all $t>0, x \in \bar{\Omega}$. By changing the origin of time to a value $t_{0}$ such that $u_{m}(x, t)>0$ for all $t \in\left[t_{0}-\tau, t_{0}\right]$ and modifying the comparison principle and (4.8) accordingly, we are assured of a comparison function $u(t)$ which tends to $\hat{u}_{m}$ as $t \rightarrow \infty$. We have proved that $u_{m}(x, t) \rightarrow \hat{u}_{m}$ as $t \rightarrow \infty$.

It remains to prove that $u_{i}(x, t) \rightarrow \hat{u}_{i}$ as $t \rightarrow \infty$. Since $u_{m}(x, t) \rightarrow \hat{u}_{m}$,

$$
b\left(u_{m}(x, t)\right)-\int_{0}^{\tau} \int_{\Omega} G(x, y, s) f(s) e^{-\gamma s} b\left(u_{m}(y, t-s)\right) d y d s \rightarrow b\left(\hat{u}_{m}\right)\left(1-\int_{0}^{\tau} f(s) e^{-\gamma s} d s\right)
$$

as $t \rightarrow \infty$, and therefore also

$$
b\left(u_{m}(x, t)\right)-\int_{0}^{\tau} \int_{\Omega} G(x, y, s) f(s) e^{-\gamma s} b\left(u_{m}(y, t-s)\right) d y d s \rightarrow \gamma \hat{u}_{i}
$$

using (3.2), and recalling that $f(s)$ has compact support here. Furthermore, the convergence in (4.9) is uniform in $x$. Thus, letting $\varepsilon>0$ be arbitrary, there exists $t_{1}>0$ such that, for all $t \geqslant t_{1}$ and all $x \in \bar{\Omega}$,

$$
b\left(u_{m}(x, t)\right)-\int_{0}^{\tau} \int_{\Omega} G(x, y, s) f(s) e^{-\gamma s} b\left(u_{m}(y, t-s)\right) d y d s \leqslant \gamma \hat{u}_{i}+\varepsilon .
$$

Then, for all $t \geqslant t_{1}$ and all $x \in \bar{\Omega}$,

$$
\frac{\partial u_{i}(x, t)}{\partial t} \leqslant D_{i} \Delta u_{i}(x, t)-\gamma u_{i}(x, t)+\gamma \hat{u}_{i}+\varepsilon
$$

By comparison, $u_{i}(x, t)$ is bounded above by the solution $\tilde{u}_{i}(x, t)$ of the linear reactiondiffusion equation

$$
\frac{\partial \tilde{u}_{i}(x, t)}{\partial t}=D_{i} \Delta \tilde{u}_{i}(x, t)-\gamma \tilde{u}_{i}(x, t)+\gamma \hat{u}_{i}+\varepsilon, \quad t>t_{1},
$$

on homogeneous Neumann boundary conditions, satisfying $\tilde{u}_{i}\left(x, t_{1}\right)=u_{i}\left(x, t_{1}\right)$ for each $x \in \bar{\Omega}$. Thus,

$$
\limsup _{t \rightarrow \infty} \max _{x \in \bar{\Omega}} u_{i}(x, t) \leqslant \hat{u}_{i}+\varepsilon / \gamma .
$$


This result holds for all $\varepsilon>0$, and therefore it holds also when $\varepsilon=0$. In a similar way, we can show that

$$
\liminf _{t \rightarrow \infty} \min _{x \in \bar{\Omega}} u_{i}(x, t) \geqslant \hat{u}_{i} .
$$

We deduce that $u_{i}(x, t) \rightarrow \hat{u}_{i}$ as $t \rightarrow \infty$, uniformly in $x$.

\subsection{Non-monotone birth functions}

The purpose of this section is to address the case when $b\left(u_{m}\right)$ qualitatively resembles $P u_{m} e^{-A u_{m}}$ (though we do not restrict to this specific example). We shall find in this case that if $\hat{u}_{m}$ exists and is in the interval of $u_{m}$ for which $b\left(u_{m}\right)$ is increasing then global convergence to $\hat{u}_{m}$ follows. If $\hat{u}_{m}$ exceeds the value of $u_{m}$ at which $b\left(u_{m}\right)$ attains its maximum then the situation is much more delicate. This case will be studied using linearised analysis. Our main assumptions for non-monotone $b\left(u_{m}\right)$ are as follows.

The kernel $f$ satisfies $f(s)=0$ for all $s \geqslant \tau$, for some $\tau>0$, and $\int_{0}^{\tau} f(s) d s=1$. Also, $b(0)=0, b\left(u_{m}\right)>0$ when $u_{m}>0, b\left(u_{m}\right)$ is differentiable and strictly increasing up to some value $u_{m}^{\max }$ and strictly de-

(A2) : $\quad$ creasing for all $u_{m}>u_{m}^{\max }$. The death function $d\left(u_{m}\right)$ is differentiable, strictly increasing for all $u_{m}>0$ and satisfies $d(0)=0$. Moreover, (3.1) has a root $\hat{u}_{m}>0$ with $b\left(u_{m}\right) \int_{0}^{\tau} f(s) e^{-\gamma s} d s>d\left(u_{m}\right)$ when $0<u_{m}<\hat{u}_{m}$ and $b\left(u_{m}\right) \int_{0}^{\tau} f(s) e^{-\gamma s} d s<d\left(u_{m}\right)$ when $u_{m}>\hat{u}_{m}$.

We will prove the following theorem.

Theorem 4 Let (A2) hold and assume that $\hat{u}_{m}<u_{m}^{\max }$. Let the initial data for system (4.1) satisfy $u_{m}^{0}(x, s) \geqslant 0$ for all $(x, s) \in \bar{\Omega} \times[-\tau, 0]$ with $u_{m}^{0}(x, 0)$ 丰 0 . Then the solution of (4.1), (4.2) satisfies

$$
\left(u_{i}(x, t), u_{m}(x, t)\right) \rightarrow\left(\hat{u}_{i}, \hat{u}_{m}\right)
$$

as $t \rightarrow \infty$, uniformly for $x \in \bar{\Omega}$, where $\hat{u}_{i}$ and $\hat{u}_{m}$ are given by (3.2) and (3.1).

Proof From the equation for $u_{m}$ in (4.1),

$$
\frac{\partial u_{m}(x, t)}{\partial t}-D_{m} \Delta u_{m}(x, t)+d\left(u_{m}(x, t)\right) \leqslant b\left(u_{m}^{\max }\right) \int_{0}^{\tau} f(s) e^{-\gamma s} d s
$$

in $\Omega \times(0, \infty)$. Well known comparison theorems for parabolic inequalities without delay (see, for example, Smith [5, Theorem 3.4]) imply that

$$
u_{m}(x, t) \leqslant w(t)
$$

where $w(t)$ satisfies

$$
w^{\prime}(t)=-d(w(t))+b\left(u_{m}^{\max }\right) \int_{0}^{\tau} f(s) e^{-\gamma s} d s
$$


subject to $w(0)=\max _{x \in \bar{\Omega}} u_{m}(x, 0)$. Therefore

$$
\limsup _{t \rightarrow \infty} \max _{x \in \bar{\Omega}} u_{m}(x, t) \leqslant d^{-1}\left(b\left(u_{m}^{\max }\right) \int_{0}^{\tau} f(s) e^{-\gamma s} d s\right) .
$$

Graphical arguments, keeping in mind the assumption $\hat{u}_{m}<u_{m}^{\max }$, can be used to show that

$$
d^{-1}\left(b\left(u_{m}^{\max }\right) \int_{0}^{\tau} f(s) e^{-\gamma s} d s\right)<u_{m}^{\max } .
$$

Let $\varepsilon>0$ be sufficiently small that

$$
d^{-1}\left(b\left(u_{m}^{\max }\right) \int_{0}^{\tau} f(s) e^{-\gamma s} d s\right)+\varepsilon<u_{m}^{\max } .
$$

There exists $T>0$ such that, for all $t \geqslant T$ and all $x \in \bar{\Omega}$,

$$
u_{m}(x, t) \leqslant d^{-1}\left(b\left(u_{m}^{\max }\right) \int_{0}^{\tau} f(s) e^{-\gamma s} d s\right)+\varepsilon .
$$

Then for all $t \geqslant T, u_{m}(x, t) \leqslant u_{m}^{\max }$, and for all $t \geqslant T+\tau$ there is no longer any record of $u_{m}(x, t)$ ever having assumed values above $u_{m}^{\max }$. The proof that $u_{m}(x, t) \rightarrow \hat{u}_{m}$ now proceeds as if the birth function $b\left(u_{m}\right)$ were increasing for all $u_{m}$, and so the result follows from Theorem 3. The proof that $u_{i}(x, t) \rightarrow \hat{u}_{i}$ is the same as for Theorem 3 .

In the rest of this section we shall examine the case when $\hat{u}_{m}>u_{m}^{\max }$. This case is much harder to study and so we shall confine ourselves to some linearised analysis in order to gain some insight into parameter regimes in which $\hat{u}_{m}$ might cease to be a stable equilibrium of the second equation of (4.1).

We shall assume (A2) holds, and that $\hat{u}_{m}>u_{m}^{\max }$. Let $\left(\mu_{k}, \phi_{k}(x)\right), k=0,1,2, \ldots$, be the eigenvalues and (normalised) eigenfunctions of $-\Delta$ on $\Omega$ with homogeneous Neumann boundary conditions. Then it is easy to see that

$$
G(x, y, t)=\sum_{k=0}^{\infty} e^{-D_{i} \mu_{k} t} \phi_{k}(x) \phi_{k}(y)
$$

and that

$$
\int_{\Omega} G(x, y, s) \phi_{k}(y) d y=e^{-D_{i} \mu_{k} s} \phi_{k}(x) .
$$

Now let $u_{m}(x, t)=\hat{u}_{m}+v(x, t)$ where $v$ is small. Substituting into the second equation of (4.1) and linearising, yields a linear equation for $v(x, t)$ which has solutions of the form $v=e^{\sigma t} \phi_{k}(x)$ whenever

$$
\sigma=-D_{m} \mu_{k}+b^{\prime}\left(\hat{u}_{m}\right) \int_{0}^{\tau} f(s) e^{-\left(\gamma+\sigma+D_{i} \mu_{k}\right) s} d s-d^{\prime}\left(\hat{u}_{m}\right) .
$$

We will analyse (4.11) under assumption (A2) with $\hat{u}_{m}>u_{m}^{\max }$. These assumptions force $b^{\prime}\left(\hat{u}_{m}\right)<0$. The structure of (4.11) enables us to apply a simple technique that leads an easily verifiable sufficient condition for all the roots $\sigma$ of (4.11) to satisfy $\operatorname{Re} \sigma<0$ for 
all $k=0,1,2, \ldots$ (i.e. for the equilibrium $\hat{u}_{m}$ of the second equation of (4.1) to be linearly stable to arbitrary small perturbations). We assume there exists a root $\sigma^{*}$ of (4.11) with $\operatorname{Re} \sigma^{*} \geqslant 0$ and proceed towards a contradiction. If such a root exists, then

$$
\begin{aligned}
\left|\sigma^{*}+D_{m} \mu_{k}+d^{\prime}\left(\hat{u}_{m}\right)\right| & \leqslant\left|b^{\prime}\left(\hat{u}_{m}\right)\right| \int_{0}^{\tau} f(s) e^{-\left(\gamma+D_{i} \mu_{k}\right) s} e^{-\left(\operatorname{Re} \sigma^{*}\right) s} d s \\
& \leqslant\left|b^{\prime}\left(\hat{u}_{m}\right)\right| \int_{0}^{\tau} f(s) e^{-\left(\gamma+D_{i} \mu_{k}\right) s} d s
\end{aligned}
$$

and it is easily seen that a contradiction is reached if

$$
\left|b^{\prime}\left(\hat{u}_{m}\right)\right| \int_{0}^{\tau} f(s) e^{-\left(\gamma+D_{i} \mu_{k}\right) s} d s<D_{m} \mu_{k}+d^{\prime}\left(\hat{u}_{m}\right)
$$

Thus, if (4.12) holds for all $k=0,1,2, \ldots$, then $\hat{u}_{m}$ is linearly stable to arbitrary small perturbations. But the eigenvalues $\mu_{k}$ of $-\Delta$ on homogeneous Neumann boundary conditions satisfy $0=\mu_{0}<\mu_{1}<\mu_{2}<\cdots$. Thus it is enough to require (4.12) to hold for $k=0$. We have proved.

Theorem 5 Let assumption (A2) hold, with $\hat{u}_{m}>u_{m}^{\max }$, and assume further that

$$
\left|b^{\prime}\left(\hat{u}_{m}\right)\right| \int_{0}^{\tau} f(s) e^{-\gamma s} d s<d^{\prime}\left(\hat{u}_{m}\right) .
$$

Then the equilibrium $\hat{u}_{m}$ of the second equation of (4.1) is linearly stable to arbitrary small perturbations.

One situation in which (4.13) will hold is if $\hat{u}_{m}$ is not much larger than $u_{m}^{\max }$, since then $\left|b^{\prime}\left(\hat{u}_{m}\right)\right|$ will be close to zero. Another situation in which (4.13) holds is if there is significant juvenile mortality (large $\gamma$ ), but one must remember that the feasibility of the equilibrium $\hat{u}_{m}$ (i.e. strict positivity of $\hat{u}_{m}$ ) depends on $\gamma$. We can show that $\hat{u}_{m}$ decreases as $\gamma$ increases, and that $\hat{u}_{m}$ may lose feasibility at a finite value of $\gamma$, or may remain feasible for all $\gamma$ (e.g. if the birth function is linear and the death function quadratic at small $u_{m}$, as in (1.1)). Naturally, the question arises as to whether $\hat{u}_{m}$ might lose stability while still feasible, leading to new dynamical behaviour in the model. To investigate this possibility, let us choose $f(s)=\delta(s-\tau)$ (the choice appropriate for situations in which all individuals take time $\tau$ to become mature), and $b\left(u_{m}\right)=P u_{m} e^{-A u_{m}}, d\left(u_{m}\right)=d_{m} u_{m}$ with $P, A$ and $d_{m}$ positive constants. In this case, $\hat{u}_{m}$ is given explicitly by

$$
\hat{u}_{m}=\frac{1}{A} \ln \left(\frac{P e^{-\gamma \tau}}{d_{m}}\right)
$$

and is feasible only for $P e^{-\gamma \tau}>d_{m}$, i.e. only up to a finite value of the juvenile mortality parameter $\gamma$. 
For simplicity, we shall look at the eigenvalue equation (4.11) corresponding to spatially uniform perturbations (i.e. we take $k=0$ ), then

$$
\sigma+d_{m}=d_{m} e^{-\sigma \tau}\left(1-\ln \left(\frac{P e^{-\gamma \tau}}{d_{m}}\right)\right) .
$$

We are thus effectively studying the dynamics of the equation

$$
u_{m}^{\prime}(t)=e^{-\gamma \tau} P u_{m}(t-\tau) e^{-A u_{m}(t-\tau)}-d_{m} u_{m}(t),
$$

a slightly different version of which was considered in Gurney et al. [2] as a model of the Australian sheep-blowfly lucilia cuprina. The quantity $P$ is the maximum rate of egg laying per adult fly. Adult blowflies live 2 to 3 weeks and typically during this time lay 2 or 3 batches of eggs containing around 200 eggs per batch. This leads to a value $P \approx 28.6$ eggs per adult per day. The maturation delay $\tau$ for blowflies in Australia is typically about 14 days. The parameter $d_{m}$ (adult mortality) is about 0.057 . The parameter $\gamma$ measures juvenile mortality (mortality during the larval and pupa stages); its value is rather more difficult to estimate but we have taken a value $\gamma \approx 0.05$ which implies a through-stage (egg to adult) survival probability of about 0.5. With these values for the parameters, the eigenvalue equation (4.14) has a pair of complex conjugate roots $0.029 \pm 0.149 i$. These eigenvalues predict a slowly growing oscillatory solution of the linearised equations and thus we are led to expect a finite amplitude limit cycle of the nonlinear equation of period approximately 42.2 days. This is in good agreement with the dynamics of laboratory populations as reported in Gurney et al. [2].

\section{Discussion}

We have proposed a simple model for a single species population incorporating stage structure and, in our opinion, a suitable means of dealing with situations in which the maturation time is not always the same for all individuals. If the birth function $b\left(u_{m}\right)$ is always increasing in $u_{m}$ and a positive equilibrium exists in ecologically reasonable circumstances (by which we mean, essentially, that adult recruitment outnumbers deaths at low densities while the opposite is true at high densities) then, regardless of the probability distribution function $f(s)$ representing the likelihood of taking time $s$ to mature, the asymptotic behaviour of the model is to an equilibrium population depending on the distribution $f(s)$.

We have also developed a reaction-diffusion extension of our model for the case of a finite spatial domain with impermeable boundaries, and shown that as $t \rightarrow \infty$ this model exhibits the dynamics described above if $b\left(u_{m}\right)$ is increasing for all $u_{m}>0$. The case of a birth function $b\left(u_{m}\right)$ qualitatively resembling $P u_{m} e^{-A u_{m}}$ is relevant for modelling some insect populations, in which the birth rate is approximately proportional to the number of adults if the number of adults is small, but is effectively zero if there are many adults since the adults may then have to devote so much energy to finding enough food for their own survival that reproduction does not happen. See, for example, Gurney et al. [2] where this point is discussed in relation to populations of blowflies. Such a non-monotone birth function with a single hump effectively partitions parameter space into two zones. 
The first of these is for parameter values which have $\hat{u}_{m}<u_{m}^{\max }$, so that the equilibrium is in the interval of values of $u_{m}$ in which $b\left(u_{m}\right)$ is increasing. In this case the interval $\left[0, u_{m}^{\max }\right]$ attracts the solutions and the long time dynamics is the same as if $b\left(u_{m}\right)$ were increasing for all $u_{m}>0$. If $\hat{u}_{m}>u_{m}^{\max }$ then the outcome depends more sensitively on the precise parameter values. If $\hat{u}_{m}$ is not too much bigger than $u_{m}^{\max }$ (in a sense made precise by inequality (4.13)) then linear analysis predicts that solutions will still to tend to $\hat{u}_{m}$, but oscillatory dynamics are a possibility for larger $\hat{u}_{m}$.

\section{References}

[1] Aiello, W. G. and Freedman, H. I. (1990) A time-delay model of single species growth with stage structure. Math. Biosci. 101, 139-153.

[2] Gurney, W. S. C., Blythe, S. P. and Nisbet, R. M. (1980) Nicholson's blowflies revisited. Nature, 287, 17-21.

[3] Martin, R. H. and Smith, H. (1990) Abstract functional differential equations and reactiondiffusion systems. Trans. Am. Math. Soc. 321, 1-44.

[4] Mischaikow, K., Smith, H. And Thieme, H. R. (1995) Asymptotically autonomous semiflows: chain recurrence and Lyapunov functions. Trans. Ar. Math. Soc. 347, 1669-1685.

[5] Sмiтh, H. L. (1995) Monotone Dynamical Systems: An introduction to the theory of competitive and cooperative systems. American Mathematical Society.

[6] Yamada, Y. (1982) On a certain class of semilinear Volterra diffusion equations. J. Math. Anal. Appl. 88, 433-451.

[7] YamadA, Y. (1984) Asymptotic stability for some systems of semilinear Volterra diffusion equations. J. Diff. Eqn. 52, 295-326. 